\title{
GEOMETRIC VERSION OF MIXED MEAN INEQUALITIES
}

\author{
YUAN JUN AND LI AIJUN
}

\begin{abstract}
In this paper, we introduce the mixed mean of star bodies and give geometric version of mixed mean inequalities.
\end{abstract}

\section{Introduction}

The classical arithmetic-geometric-harmonic mean inequality is one of the most important analytic inequalities, which is used in almost every branch of mathematics. There is a huge amount of work on its generalization (see [1, 2, 3, 4, 5], [7, 8, 9, 10, 11, 12, 13]).

In this paper, we introduce several kinds of mixed means for star bodies, which involve the geometric mean and one of the arithmetic and harmonic means, and prove some related mixed mean inequalities.

For star bodies $K, L$, let $K \widetilde{+} L$ and $K L$ denote the radial addition and multiplication of $K$ and $L$, respectively. Our result is the following theorem, which is a special case of Theorem 4.1 of this paper:

Theorem 1. Let the arithmetic and geometric means of the star bodies $K_{1}, K_{2}, \ldots$, $K_{n}$ taken $n-1$ at a time be denoted by

$$
A_{i}=\frac{K_{1} \tilde{+} \cdots K_{i-1} \tilde{+} K_{i+1} \tilde{+} \cdots \widetilde{+} K_{n}}{n-1}, \quad G_{i}=\left(K_{1} \cdots K_{i-1} K_{i+1} \cdots K_{n}\right)^{\frac{1}{n-1}} .
$$

Then for $n \geq 3$;

$$
\left(A_{1} \cdots A_{n}\right)^{\frac{1}{n}} \geq \frac{G_{1} \widetilde{+} \cdots \widetilde{+} G_{n}}{n} .
$$

with equality holds if and only if $K_{1}=K_{2}=\cdots=K_{n}$. Note that $K \geq L$ means that $K \supseteq L$.

Remark 1. The inequality of (1.1) can be viewed as a geometric version of mixed arithmetic and geometry mean inequality established in [3, 4] for positive scalars.

Corresponding author: Yuan Jun.

Received December 30, 2007.

2000 Mathematics Subject Classification. 52A20, 26D07.

Key words and phrases. Star body, star dual, geometric mean, mixed mean.

Supported in part by Jiangsu Planned Projects for Postdoctoral Research Funds (0801043C) and the National Natural Science Foundation of China. (Grant NO.10271071). 
Please see the next section for above interrelated notations and definitions.

\section{Basic Definitions and notation}

As usual, let $B_{n}$ denote the unit ball in Euclidean $n$-space, $\mathbb{R}^{n}$. While its boundary is $S^{n-1}$. For a compact set $K$ of $\mathbb{R}^{n}$, which is star-shaped with respect to the origin, define the radial function $\rho(K, \cdot)$ of $K$ by $([6],[16])$

$$
\rho(K, u)=\max \{\lambda \geq 0: \lambda u \in K\} \quad u \in S^{n-1} .
$$

If $\rho(K, \cdot)$ is continuous, $K$ will be called a star body. Let $\mathcal{S}^{n}$ denote the set of star bodies in $\mathbb{R}^{n}$.

From the definition of radial function it follows that if $K, L \in \mathcal{S}^{n},[6]$

$$
K \geq L \Leftrightarrow \rho(K, u) \geq \rho(L, u)
$$

for all $u \in S^{n-1}$.

If $x_{i} \in \mathbb{R}^{n}, 1 \leq i \leq m$, then $x_{1} \tilde{+} \ldots \tilde{+} x_{m}$ is defined to be the usual vector sum of the points $x_{i}$, if all of them are contained in a line though $o$, and 0 otherwise.

Let $K_{i} \in \mathcal{S}^{n}$, and $t_{i} \geq 0,1 \leq i \leq m$, then

$$
t_{1} K_{1} \tilde{+} \ldots \tilde{+} t_{m} K_{m}=\left\{t_{1} x_{1} \tilde{+} \ldots \tilde{+} t_{m} x_{m}: x_{i} \in K_{i}\right\}
$$

is called a radial linear combination. The addition and scalar multiplication are called radial addition and radial scalar multiplication. Moreover,

$$
\rho\left(t_{1} K_{1} \tilde{+} t_{2} K_{2}, u\right)=t_{1} \rho\left(K_{1}, u\right)+t_{2} \rho\left(K_{2}, u\right),
$$

for all $u \in S^{n-1}$.

Also associated with a star body $K \in \mathcal{S}^{n}$ is its star dual $K^{\circ}$, which was introduced by Moszyńska [14] (and was improved in [15]). Let $i$ be the inversion of $\mathbb{R}^{n} \backslash\{0\}$, with respect to $S^{n-1}$ :

$$
i(x):=\frac{x}{\|x\|^{2}} .
$$

Then the star dual $K^{\circ}$ of a star body $K \in \mathcal{S}^{n}$ is defined by

$$
K^{\circ}=\operatorname{cl}\left(\mathbb{R}^{n} \backslash i(L)\right) .
$$

It is easy to verify that for every $u \in S^{n-1}[14]$,

$$
\rho\left(K^{\circ}, u\right)=\frac{1}{\rho(K, u)},
$$

and

$$
K^{\circ \circ}=K
$$


Moreover, for $K, L \in \mathcal{S}^{n}$,

$$
K \geq L \Leftrightarrow K^{\circ} \leq L^{\circ}
$$

Let $K_{1}, \ldots, K_{n} \in \mathcal{S}^{n}(n \geq 2)$. We define the arithmetic mean of $K_{1}, \ldots, K_{n}$ as

$$
\mathbf{A}\left(K_{1}, \ldots, K_{n}\right):=\frac{K_{1} \tilde{+} \cdots \tilde{+} K_{n}}{n}=\frac{1}{n} \sum_{i=1}^{n} K_{i}
$$

and the harmonic mean of $K_{1}, \ldots, K_{n}$ as

$$
\mathbf{H}\left(K_{1}, \ldots, K_{n}\right):=\left(\frac{K_{1}^{\circ} \tilde{+} \cdots \widetilde{+} K_{n}^{\circ}}{n}\right)^{\circ}=\left(\frac{1}{n} \sum_{i=1}^{n} K_{i}^{\circ}\right)^{\circ}
$$

Throughout the paper, for notation $\sum_{i}^{n} X_{i}$, if $X_{i}$ is a star body, then the $\sum$ denotes the radial addition, and usual sum otherwise.

\section{Geometric mean inequalities for star bodies}

Let $K_{i} \in \mathcal{S}^{n}(1 \leq i \leq n)$. The multiplication, $K_{1} \cdots K_{n}$, of $K_{1}, \ldots, K_{n}$ is a star body whose radial function satisfies for $u \in S^{n-1}$,

$$
\rho\left(K_{1} \cdots K_{n}, u\right)=\rho\left(K_{1}, u\right) \rho\left(K_{2}, u\right) \cdots \rho\left(K_{n}, u\right) .
$$

The geometric mean, $\mathbf{G}\left(K_{1}, \ldots, K_{n}\right)$, of $K_{1}, \ldots, K_{n}$ is a star body whose radial function satisfies for $u \in S^{n-1}$,

$$
\rho\left(\mathbf{G}\left(K_{1}, \ldots, K_{n}\right), u\right)=\left[\rho_{K_{1}}(u) \rho_{K_{2}}(u) \cdots \rho_{K_{n}}(u)\right]^{\frac{1}{n}}
$$

From the definition of radial function it follows that $\mathbf{G}\left(K_{1}, \ldots, K_{n}\right)$ is symmetric in its arguments. It also follows that $\mathbf{G}(K, \ldots, K)=K$. We use the notation $\mathbf{G}(\underbrace{K, \ldots, K}_{p}, \underbrace{L, \ldots, L}_{n-p})$ with $p$ copies of $K$ and $n-p$ copies of $L$.

Now, we develop some basic properties of the $\mathbf{G}\left(K_{1}, \ldots, K_{n}\right)$ which are useful in our discussion.

Lemma 3.1. Let $K_{i}, L_{i} \in \mathcal{S}^{n}(1 \leq i \leq n)$ and $\alpha, \beta>0$. Then

$$
\begin{aligned}
& \mathbf{G}\left(\alpha K_{1} \tilde{+} \beta L_{1}, \ldots, \alpha K_{n} \tilde{+} \beta L_{n}\right) \\
& \quad \geq \alpha \mathbf{G}\left(K_{1}, \ldots, K_{n}\right) \tilde{+} \beta \mathbf{G}\left(L_{1}, \ldots, L_{n}\right) .
\end{aligned}
$$


Proof. For every $u \in S^{n-1}$, by (2.3), (3.1) and Maclaurin's inequality, we have

$$
\begin{aligned}
\rho(\mathbf{G} & \left.\left(\alpha K_{1} \tilde{+} \beta L_{1}, \ldots, \alpha K_{n} \tilde{+} \beta L_{n}\right), u\right) \\
& =\left(\prod_{i=1}^{n} \alpha \rho\left(K_{i}, u\right)+\beta \rho\left(L_{i}, u\right)\right)^{\frac{1}{n}} \\
& \geq \alpha \prod_{i=1}^{n} \rho\left(K_{i}, u\right)^{\frac{1}{n}}+\beta \prod_{i=1}^{n} \rho\left(L_{i}, u\right)^{\frac{1}{n}} \\
& =\rho\left(\alpha \mathbf{G}\left(K_{1}, \ldots, K_{n}\right) \tilde{+} \beta \mathbf{G}\left(L_{1}, \ldots, L_{n}\right), u\right) .
\end{aligned}
$$

By (2.2), we complete the proof of the lemma.

Lemma 3.1. and the mathematical induction yield immediately the following property of $\mathbf{G}\left(K_{1}, \ldots, K_{n}\right)$.

Lemma 3.2. Let $K_{i j} \in \mathcal{S}^{n},(1 \leq i \leq n, 1 \leq j \leq m)$. Then

$$
\mathbf{G}\left(\sum_{j=1}^{m} K_{1 j}, \ldots, \sum_{j=1}^{m} K_{n j}\right) \geq \sum_{j=1}^{m} \mathbf{G}\left(K_{1 j}, \ldots, K_{n j}\right)
$$

In lemma 3.2 , if we choose $m=n-1$ and $\left(K_{i 1}, \ldots, K_{i m}\right)=\left(K_{j}\right)_{j \neq i}$ for given star bodies $K_{1}, \ldots, K_{n}$. We get

Lemma 3.3. Let $K_{1}, \ldots, K_{n} \in \mathcal{S}^{n},(n \geq 3)$. Then

$$
\mathbf{G}\left(\sum_{i \neq 1} K_{i}, \ldots, \sum_{i \neq n} K_{i}\right) \geq \frac{2}{n(n-1)} \sum_{1 \leq i<j \leq n} \sum_{k=1}^{n-1} \mathbf{G}(\underbrace{K_{i}, \ldots, K_{i}}_{k}, \underbrace{K_{j}, \ldots, K_{j}}_{n-k}) .
$$

Proof. Let $\pi$ be an arbitrary permutation of $(1, \ldots, n)$. By symmetry and Lemma 3.2 , we have

$$
\begin{aligned}
\mathbf{G}\left(\sum_{i \neq 1} K_{i}, \ldots, \sum_{i \neq n} K_{i}\right)= & \mathbf{G}\left(\sum_{i \neq 1} K_{\pi(i)}, \ldots, \sum_{i \neq n} K_{\pi(i)}\right) \\
\geq & \mathbf{G}\left(K_{\pi(2)}, K_{\pi(1)}, \ldots, K_{\pi(1)}\right) \\
& +\mathbf{G}\left(K_{\pi(3)}, K_{\pi(3)}, K_{\pi(2)}, \ldots, K_{\pi(2)}\right) \\
& +\cdots+\mathbf{G}\left(K_{\pi(n)}, \ldots, K_{\pi(n)}, K_{\pi(n-1)}\right)
\end{aligned}
$$


Since the number of all permutations of $(1, \ldots, n)$ is $n$ !,

$$
\begin{aligned}
n ! \mathbf{G}\left(\sum_{i \neq 1} K_{i}, \ldots, \sum_{i \neq n} K_{i}\right) \geq & \sum_{\pi}\left\{\mathbf{G}\left(K_{\pi(2)}, K_{\pi(1)}, \ldots, K_{\pi(1)}\right)\right. \\
& +\mathbf{G}\left(K_{\pi(3)}, K_{\pi(3)}, K_{\pi(2)}, \ldots, K_{\pi(2)}\right) \\
& \left.+\cdots+\mathbf{G}\left(K_{\pi(n)}, \ldots, K_{\pi(n)}, K_{\pi(n-1)}\right)\right\} \\
= & \left.\sum_{\pi} \sum_{k=1}^{n-1} \mathbf{G}(\underbrace{K_{\pi(k+1)}, \ldots, K_{\pi(k+1)}}_{k}, \underbrace{K_{\pi(k)}, \ldots, K_{\pi(k)}}_{n-k})\right\}
\end{aligned}
$$

Note that each term

$$
\mathbf{G}(\underbrace{K_{i}, \ldots, K_{i}}_{k}, \underbrace{K_{j}, \ldots, K_{j}}_{n-k}) \quad(1 \leq k \leq n-1)
$$

for $i \neq j$ appears $(n-2)$ ! times in the summand of the right side of $(3.6)$, because the number of the permutations $\pi$ such that

$$
\pi(k+1)=i, \quad \pi(k)=j \quad(1 \leq k \leq n-1)
$$

for $i \neq j$ is $(n-2)$ !. Thus,

$$
\begin{aligned}
n(n-1) \mathbf{G}\left(\sum_{i \neq 1} K_{i}, \ldots, \sum_{i \neq n} K_{i}\right) & \geq \sum_{i \neq j} \sum_{k=1}^{n-1} \mathbf{G}(\underbrace{K_{i}, \ldots, K_{i}}_{k}, \underbrace{K_{j}, \ldots, K_{j}}_{n-k}) \\
& =2 \sum_{1 \leq i<j \leq n} \sum_{k=1}^{n-1} \mathbf{G}(\underbrace{K_{i}, \ldots, K_{i}}_{k}, \underbrace{K_{j}, \ldots, K_{j}}_{n-k}) .
\end{aligned}
$$

From the last equation we deduce immediately the inequality (3.5).

The following lemma gives an upper bound of $\mathbf{G}\left(K_{1}, \ldots, K_{n}\right)$ in terms of $\mathbf{G}\left(K_{i}, K_{j}\right)$ $(i \neq j)$.

Lemma 3.4. Let $K_{1}, \ldots, K_{n} \in \mathcal{S}^{n},(n \geq 3)$. Then

$$
\mathbf{G}\left(K_{1}, \ldots, K_{n}\right) \leq \frac{2}{n(n-1)} \sum_{1 \leq i<j \leq n} \mathbf{G}\left(K_{i}, K_{j}\right)
$$


Proof. For every $u \in S^{n-1}$,

$$
\begin{aligned}
\rho\left(\frac{2}{n(n-1)} \sum_{1 \leq i<j \leq n} \mathbf{G}\left(K_{i}, K_{j}\right), u\right) & =\frac{2}{n(n-1)} \sum_{1 \leq i<j \leq n} \rho\left(\mathbf{G}\left(K_{i}, K_{j}\right), u\right) \\
& =\frac{2}{n(n-1)} \sum_{1 \leq i<j \leq n} \rho\left(K_{i}, u\right)^{\frac{1}{2}} \rho\left(K_{j}, u\right)^{\frac{1}{2}} \\
& \geq\left[\prod_{1 \leq i<j \leq n} \rho\left(K_{i}, u\right)^{\frac{1}{2}} \rho\left(K_{j}, u\right)^{\frac{1}{2}}\right]^{\frac{2}{n(n-1)}} \\
& =\rho\left(\mathbf{G}\left(K_{1}, \ldots, K_{n}\right), u\right)
\end{aligned}
$$

This complete the proof of the lemma.

Lemma 3.5. Let $K_{1}, \ldots, K_{n} \in \mathcal{S}^{n},(n \geq 3)$. Then

$$
\mathbf{A}\left(K_{1}, \ldots, K_{n}\right) \geq \mathbf{G}\left(K_{1}, \ldots, K_{n}\right) \geq \mathbf{H}\left(K_{1}, \ldots, K_{n}\right) .
$$

Proof. For every $u \in S^{n-1}$, by (2.6), (3.1) and (2.4), we have

$$
\begin{aligned}
\rho\left(\mathbf{A}\left(K_{1}, \ldots, K_{n}\right), u\right) & =\frac{\rho\left(K_{1}, u\right)+\rho\left(K_{2}, u\right)+\cdots+\rho\left(K_{n}, u\right)}{n} \\
& \geq\left(\rho\left(K_{1}, u\right) \rho\left(K_{1}, u\right) \cdots \rho\left(K_{n}, u\right)\right)^{\frac{1}{n}} \\
& =\rho\left(\mathbf{G}\left(K_{1}, \ldots, K_{n}\right), u\right) \\
& \geq \frac{n}{\rho\left(K_{1}^{\circ}, u\right)+\rho\left(K_{2}^{\circ}, u\right)+\cdots+\rho\left(K_{n}^{\circ}, u\right)} \\
& =\rho\left(\mathbf{H}\left(K_{1}^{\circ}, \ldots, K_{n}^{\circ}\right), u\right) .
\end{aligned}
$$

This complete the proof of the lemma.

\section{Mixed mean inequalities for star bodies}

In this section, we turn our attention of four types of mixed means for star bodies, and derive some related star body mean inequalities.

Let $K_{1}, \ldots, K_{n} \in \mathcal{S}^{n},(n \geq 3)$. We introduce the following four types of mixed means:

(1) $\widetilde{\mathbf{A}}\left(K_{1}, \ldots, K_{n}\right):=\mathbf{A}\left(\mathbf{G}\left(\left(K_{i}\right)_{i \neq 1}\right), \mathbf{G}\left(\left(K_{i}\right)_{i \neq 2}\right), \ldots, \mathbf{G}\left(\left(K_{i}\right)_{i \neq n}\right)\right)$;

(2) $\widetilde{\mathbf{G}}\left(K_{1}, \ldots, K_{n}\right):=\mathbf{G}\left(\mathbf{A}\left(\left(K_{i}\right)_{i \neq 1}\right), \mathbf{A}\left(\left(K_{i}\right)_{i \neq 2}\right), \ldots, \mathbf{A}\left(\left(K_{i}\right)_{i \neq n}\right)\right)$;

(3) $\widehat{\mathbf{G}}\left(K_{1}, \ldots, K_{n}\right):=\mathbf{G}\left(\mathbf{H}\left(\left(K_{i}\right)_{i \neq 1}\right), \mathbf{H}\left(\left(K_{i}\right)_{i \neq 2}\right), \ldots, \mathbf{H}\left(\left(K_{i}\right)_{i \neq n}\right)\right)$;

(4) $\hat{\mathbf{H}}\left(K_{1}, \ldots, K_{n}\right):=\mathbf{H}\left(\mathbf{G}\left(\left(K_{i}\right)_{i \neq 1}\right), \mathbf{G}\left(\left(K_{i}\right)_{i \neq 2}\right), \ldots, \mathbf{G}\left(\left(K_{i}\right)_{i \neq n}\right)\right)$;

The following main theorem refines upon the star bodies arithmetic-geometric mean inequality given in (3.8). 
Theorem 4.1. Let $K_{i} \in \mathcal{S}^{n}(1 \leq i \leq n)$. Then

$$
\mathbf{A}\left(K_{1}, \ldots, K_{n}\right) \geq \widetilde{\mathbf{G}}\left(K_{1}, \ldots, K_{n}\right) \geq \widetilde{\mathbf{A}}\left(K_{1}, \ldots, K_{n}\right) \geq \mathbf{G}\left(K_{1}, \ldots, K_{n}\right) .
$$

Proof. Observe from (3.2) and (3.8) that

$$
\begin{aligned}
\widetilde{\mathbf{A}}\left(K_{1}, \ldots, K_{n}\right) & =\frac{\mathbf{G}\left(\left(K_{i}\right)_{i \neq 1}\right) \tilde{+} \mathbf{G}\left(\left(K_{i}\right)_{i \neq 2}\right) \tilde{+} \cdots \tilde{+} \mathbf{G}\left(\left(K_{i}\right)_{i \neq n}\right)}{n} \\
& \geq \mathbf{G}\left(\mathbf{G}\left(\left(K_{i}\right)_{i \neq 1}\right), \mathbf{G}\left(\left(K_{i}\right)_{i \neq 2}\right), \ldots, \mathbf{G}\left(\left(K_{i}\right)_{i \neq n}\right)\right) \\
& =\mathbf{G}\left(K_{1}, \ldots, K_{n}\right) .
\end{aligned}
$$

Then the last inequality of (4.1) holds.

Furthermore, by the first inequality of (3.8), we get

$$
\begin{aligned}
\widetilde{\mathbf{G}}\left(K_{1}, \ldots, K_{n}\right) & \leq \frac{\mathbf{A}\left(\left(K_{i}\right)_{i \neq 1}\right) \tilde{+} \mathbf{A}\left(\left(K_{i}\right)_{i \neq 2}\right) \tilde{+} \ldots \tilde{+} \mathbf{A}\left(\left(K_{i}\right)_{i \neq n}\right)}{n} \\
& =\frac{K_{1} \tilde{+} K_{2} \tilde{+} \cdots \tilde{+} K_{n}}{n}=\mathbf{A}\left(K_{1}, \ldots, K_{n}\right) .
\end{aligned}
$$

This proves the first inequality of (4.1).

Now it remains to prove

$$
\widetilde{\mathbf{G}}\left(K_{1}, \ldots, K_{n}\right) \geq \widetilde{\mathbf{A}}\left(K_{1}, \ldots, K_{n}\right) .
$$

Application of Lemma 3.4 yields

$$
\begin{aligned}
\widetilde{\mathbf{A}}\left(K_{1}, \ldots, K_{n}\right) & =\frac{\mathbf{G}\left(\left(K_{i}\right)_{i \neq 1}\right) \tilde{+} \mathbf{G}\left(\left(K_{i}\right)_{i \neq 2}\right) \tilde{+} \ldots \tilde{+} \mathbf{G}\left(\left(K_{i}\right)_{i \neq n}\right)}{n} \\
& \leq \frac{2}{n(n-1)(n-2)}\left(\sum_{\substack{1 \leq i<j \leq n \\
i, j \neq 1}} \mathbf{G}\left(K_{i}, K_{j}\right) \tilde{+} \ldots \widetilde{+} \sum_{\substack{1 \leq i<j \leq n \\
i, j \neq n}} \mathbf{G}\left(K_{i}, K_{j}\right)\right) \\
& =\frac{2}{n(n-1)} \sum_{1 \leq i<j \leq n} \mathbf{G}\left(K_{i}, K_{j}\right) .
\end{aligned}
$$

On the other hand, from Lemma 3.3 we obtain

$$
\begin{aligned}
\widetilde{\mathbf{G}}\left(K_{1}, \ldots, K_{n}\right) & =\mathbf{G}\left(\frac{1}{n-1} \sum_{i \neq 1} K_{i}, \frac{1}{n-1} \sum_{i \neq 2} K_{i}, \ldots, \frac{1}{n-1} \sum_{i \neq n} K_{i}\right) \\
& \geq \frac{2}{n(n-1)^{2}} \sum_{1 \leq i<j \leq n} \sum_{k=1}^{n-1} \mathbf{G}(\underbrace{K_{i}, \ldots, K_{i}}_{k}, \underbrace{K_{j}, \ldots, K_{j}}_{n-k}) .
\end{aligned}
$$

Next it suffices to check that for all $i, j(i \neq j)$,

$$
\sum_{k=1}^{n-1} \mathbf{G}(\underbrace{K_{i}, \ldots, K_{i}}_{k}, \underbrace{K_{j}, \ldots, K_{j}}_{n-k}) \geq(n-1) \mathbf{G}\left(K_{i}, K_{j}\right) .
$$


For every $u \in S^{n-1}$,

$$
\begin{aligned}
\rho\left(\sum_{k=1}^{n-1} \mathbf{G}(\underbrace{K_{i}, \ldots, K_{i}}_{k}, \underbrace{K_{j}, \ldots, K_{j}}_{n-k}), u\right) & =\sum_{k=1}^{n-1} \rho\left(\mathbf{G}(\underbrace{K_{i}, \ldots, K_{i}}_{k}, \underbrace{K_{j}, \ldots, K_{j}}_{n-k}), u\right) \\
& =\sum_{k=1}^{n-1} \rho\left(K_{i}, u\right)^{\frac{k}{n}} \rho\left(K_{j}, u\right)^{\frac{n-k}{n}} \\
& \geq(n-1) \prod_{k=1}^{n-1} \rho\left(K_{i}, u\right)^{\frac{k}{n(n-1)}} \rho\left(K_{j}, u\right)^{\frac{n-k}{n(n-1)}} \\
& =(n-1) \rho\left(\mathbf{G}\left(K_{i}, K_{j}\right), u\right) .
\end{aligned}
$$

Hence the inequality (4.5) is verified.

From (4.3), (4.4) and (4.5), we infer finally the inequality (4.2). Theorem 4.1 is therefore proved.

Replacing $K_{i}$ by $K_{i}^{\circ}(1 \leq i \leq n)$ in (4.1) and taking the star dual of each term, we arrive at the following mean inequality chain, which refines upon the star bodies harmonic-geometric mean inequality given in (3.8)

Theorem 4.2. Let $K_{i} \in \mathcal{S}^{n}(1 \leq i \leq n)$. Then

$$
\mathbf{G}\left(K_{1}, \ldots, K_{n}\right) \geq \widehat{\mathbf{H}}\left(K_{1}, \ldots, K_{n}\right) \geq \widehat{\mathbf{G}}\left(K_{1}, \ldots, K_{n}\right) \geq \mathbf{H}\left(K_{1}, \ldots, K_{n}\right) .
$$

Proof. Replacing each $K_{i}$ by its star dual in (4.1), we obtain

$$
\mathbf{A}\left(K_{1}^{\circ}, \ldots, K_{n}^{\circ}\right) \geq \widetilde{\mathbf{G}}\left(K_{1}^{\circ}, \ldots, K_{n}^{\circ}\right) \geq \widetilde{\mathbf{A}}\left(K_{1}^{\circ}, \ldots, K_{n}^{\circ}\right) \geq \mathbf{G}\left(K_{1}^{\circ}, \ldots, K_{n}^{\circ}\right) .
$$

and therefore

$$
\mathbf{G}\left(K_{1}^{\circ}, \ldots, K_{n}^{\circ}\right)^{\circ} \geq \widetilde{\mathbf{A}}\left(K_{1}^{\circ}, \ldots, K_{n}^{\circ}\right)^{\circ} \geq \widetilde{\mathbf{G}}\left(K_{1}^{\circ}, \ldots, K_{n}^{\circ}\right)^{\circ} \geq \mathbf{A}\left(K_{1}^{\circ}, \ldots, K_{n}^{\circ}\right)^{\circ} .
$$

From (2.5), we check easily that the following relations hold:

$$
\begin{aligned}
\mathbf{H}\left(K_{1}, \ldots, K_{n}\right) & =\mathbf{A}\left(K_{1}^{\circ}, \ldots, K_{n}^{\circ}\right)^{\circ}, \\
\mathbf{G}\left(K_{1}, \ldots, K_{n}\right) & =\mathbf{G}\left(K_{1}^{\circ}, \ldots, K_{n}^{\circ}\right)^{\circ}, \\
\widehat{\mathbf{H}}\left(K_{1}, \ldots, K_{n}\right) & =\widetilde{\mathbf{A}}\left(K_{1}^{\circ}, \ldots, K_{n}^{\circ}\right)^{\circ}, \\
\widehat{\mathbf{G}}\left(K_{1}, \ldots, K_{n}\right) & =\widetilde{\mathbf{G}}\left(K_{1}^{\circ}, \ldots, K_{n}^{\circ}\right)^{\circ} .
\end{aligned}
$$

Inserting (4.8) into (4.7), we get immediately (4.6). Thus we complete the proof of Theorem 4.2. 


\section{References}

[1] M. Alić, B. Mond, J. Pečarić and V. Volenec, The Arithmetic-Geometric-Harmonic-Mean and Related Matrix Inequalities, Linear Algebra Appl., 264 (1997), 55-62.

[2] E. F. Beckenbach and R. Bellman, Inequalities, Springer, Berlin, 1961.

[3] B. C. Carlson, R. K. Meany and S. A. Nelson, An inequality of mixed arithmetic and geometric means, SIAM Rev., 13 (1971), 253-255.

[4] B. C. Carlson, R. K. Meany and S. A. Nelson, Mixed arithmetic and geometric means, Pacific J. Math., 38 (1971), 343-349.

[5] H. Z. Chuan, Note on the inequality of the arithmetic and geometric means, Pacific J.Math. 143, (1990), 43-46.

[6] R.J. Gardner, Geometric Tomography, Cambridge University Press, New York, 1995.

[7] G. H. Hardy, J. E. Littlewood and G. Pólya, Inequalities, second edition, Cambridge University Press, Cambridge, 1952.

[8] Y. J. Hu, X. P. Zhang and Z. H. Yang, Mixed mean inequalities for several positive definite matrices, Linear Algebra Appl. 395 (2005), 247-263.

[9] H. Kober, On the arithmetic and geometric means and on Hölder's inequality, Proc. Amer. Math. Soc., 9 (1958), 452-459.

[10] G. S. Leng, L. Si, and Q. S. Zhu, Mixed-Mean Inequalities for Subsets, Proc. Amer. Math. Soc., 132 (2004), 2655-2660.

[11] B. Mond, J. Pečarić, A mixed arithmetic-mean harmonic-mean matrix inequality, Linear Algebra Appl. 237/238 (1996), 449-454.

[12] D. S. Mitrinović, Analytic inequalities, Springer, New York, 1970.

[13] D. S. Mitrinović, J. E. Pečarić and A. M. Fink, Classical and New Inequalities in Analysis, Kluwer, Dordrecht, 1993.

[14] M. Moszyńska, Quotient Star Bodies, Intersection Bodies and Star Duality, J. Math. Anal. Appl. 232(1999), 45-60.

[15] M. Moszyńska, Selected Topics in Convex Geometry, Springer Verlag, 2005.

[16] R. Schneider, Convex Bodies: The Brunn-Minkowski Theory, Cambridge University Press, Cambridge, 1993.

School of Mathematics and Computer Science, Nanjing Normal University, Nanjing, 210097, P.R.China

E-mail: yuanjun_math@126.com

Department of Mathematics, Shanghai University, Shanghai, 200444, P.R.China.

E-mail: liaijun72@163.com 